\title{
CLINICAL ASSESSMENT OF A HAND-HELD AUTOMATED KERATOMETER IN CATARACT SURGERY
}

\author{
MARTIN LEYLAND and LARRY BENJAMIN \\ Aylesbury
}

\begin{abstract}
SUMMARY
Purpose: Pre-operative keratometry was performed on 32 eyes of 32 patients undergoing extracapsular cataract extraction with intraocular lens implantation, for calculation of intraocular lens power. In an additional 20 eyes of 20 patients post-operative keratometry was performed to guide selective suture removal. Readings from a manual keratometer and an automated handheld keratometer were compared.

Methods: Pre-operative measurements were repeated three times on each subject to assess the repeatability of each machine. Mean-difference plots were performed to define the limits of agreement of the two machines. Results: Repeatability was higher using manual keratometry (MK) than automated keratometry (AK). There was broad agreement between the two machines in pre-operative and post-operative assessment, although clinically significant differences are likely to occur in some cases.

Conclusions: MK should continue to be used for routine pre-operative keratometry, with the AK providing a useful alternative when MK is not possible. AK is sufficiently accurate to allow its use in post-operative assessment of suture-induced astigmatism.
\end{abstract}

Keratometry has two main uses in cataract surgery: to measure corneal radius of curvature for use in calculation of intraocular lens power ${ }^{1}$ and to assess the axis and magnitude of any suture-induced astigmatism. ${ }^{2}$ The most commonly used keratometer in the UK uses the Javal-Schiotz principle, observing the Purkinje-Sanson I image created by an optically doubled target of variable size. Manual keratometry requires a skilled operator and is slower than automated keratometry, ${ }^{3}$ which can be a problem with frail or unco-operative patients. A portable

From: Ophthalmic Unit, Stoke Mandeville Hospital NHS Trust, Mandeville Road, Aylesbury, Bucks HP21 8AL, UK.

Correspondence to: Mr M. Leyland, Western Eye Hospital, Marylebone Road, London NW1 5YE, UK. hand-held automated keratometer offers further advantages for use in these difficult patient groups, such as bed-bound or anaesthetised patients.

This study aimed to assess the repeatability and accuracy of a hand-held keratometer in comparison with standard manual keratometry.

\section{PATIENTS AND METHODS}

\section{Automated Keratometry (AK)}

The Alcon Auto-Keratometer (Alcon Systems, USA) was used in this study. It is a rechargeable self-contained hand-held machine that measures the size of the Purkinje-Sanson I images produced by four projected target lights. The operator assesses the correct instrument-to-patient distance from the pattern of reflected lights on the cornea. The keratometer automatically adjusts for deviations from vertical of up to $15^{\circ}$ and gives a reading of radius of curvature between 5.63 and $11.25 \mathrm{~mm}$ in $0.01 \mathrm{~mm}$ increments. A circular ring of mires allows subjective assessment of corneal distortion. No calibration is required by the user.

\section{Manual Keratometry (MK)}

The Haag-Streit Javal-Schiotz keratometer was used for manual readings, measuring radius of corneal curvature from 5.5 to. $11.0 \mathrm{~mm}$ in $0.1 \mathrm{~mm}$ increments, estimated by interpolation to $0.05 \mathrm{~mm}$. The machine was calibrated according to the manufacturer's instructions.

\section{The Study}

The study was in two parts, examining both preoperative and post-operative eyes.

Pre-operative Keratometry for Intraocular Lens Power Calculation. A prospective study was carried out on 32 patients admitted for extracapsular cataract extraction (ECCE) via a clear corneal incision and with placement of a posterior chamber intraocular 

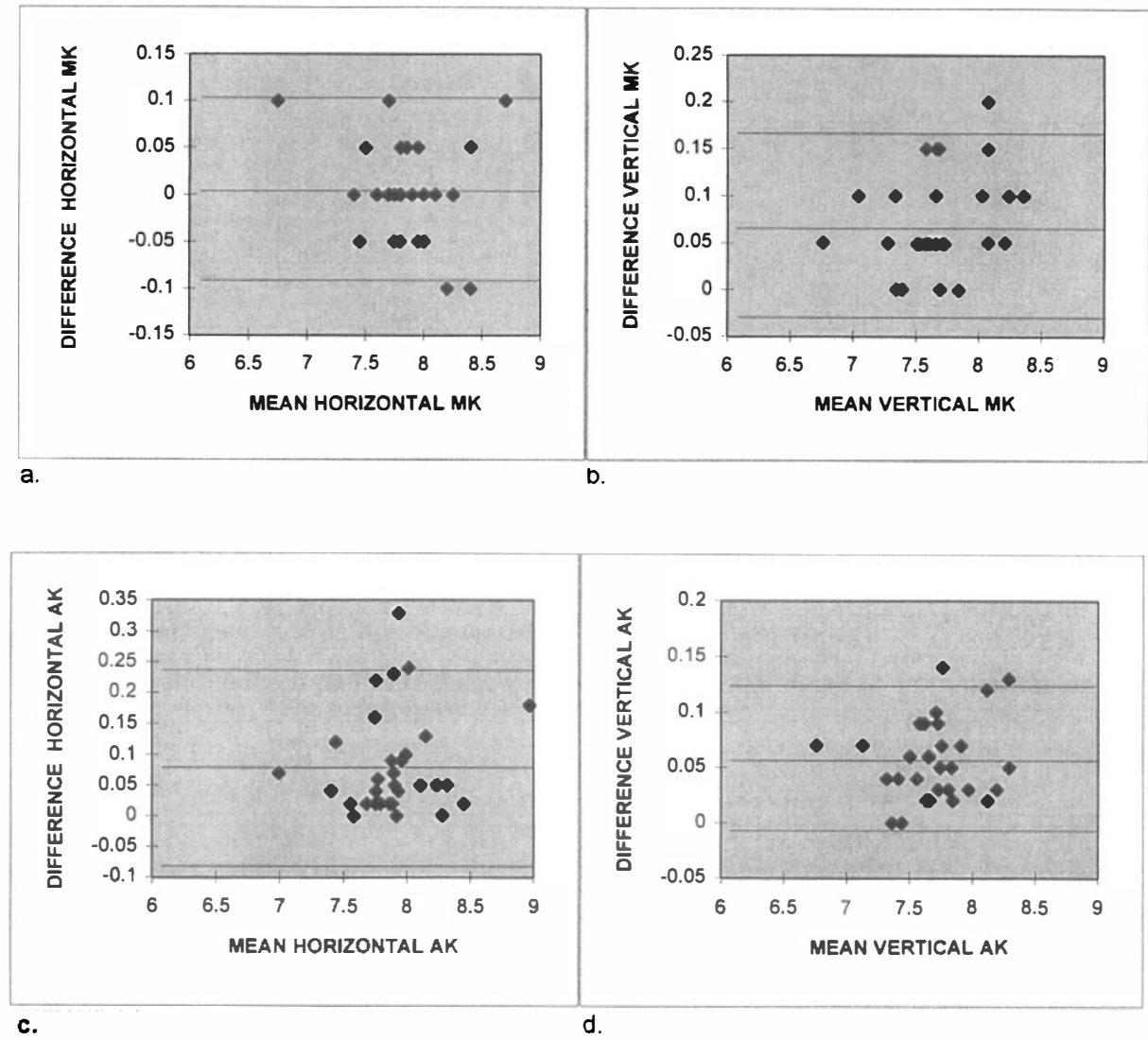

Fig. 1. Pre-operative keratometry: repeatability of $M K$ and $A K$. The horizontal lines mark the mean difference and $\pm 1.96 S D$ of the differences. (a) MK horizontal meridian. (b) $M K$ vertical meridian. (c) AK horizontal meridian. (d) AK vertical meridian.

lens (IOL). In all cases only the eye to be operated upon was measured and had no pre-existing corneal disease. Cataract surgery was performed by the consultants and resident staff at Stoke Mandeville Hospital NHS Trust, Aylesbury. Maximal and minimal corneal radii of curvature were measured; that nearest to $180^{\circ}$ was recorded as 'horizontal', and that nearest to $90^{\circ}$ as 'vertical'. Each eye was measured three times with $\mathrm{MK}$; between each reading the instrument was withdrawn and the mires randomly moved. This was followed by three readings with AK.

Post-operative Keratometry for Magnitude and Axis of Astigmatism. Twenty consecutive patients had single readings taken using MK and AK 6-16 weeks following ECCE with posterior chamber IOL via a clear corneal incision and without prior corneal disease.

\section{Analysis}

Repeatability of each instrument and the comparison of MK with $\mathrm{AK}$ on a subject were assessed using mean-difference plots as described by Bland and Altman. ${ }^{4}$ Limits of agreement were calculated for both repeatability and agreement of the two machines. In this analysis, a wide limit of agreement implies poor repeatability of a given test or poor agreement between the two machines.

\section{RESULTS}

\section{Pre-operative Keratometry}

Repeatability was assessed with a mean-difference plot using the mean of the three readings on a single eye and the difference between the largest and smallest readings (Fig. 1). The limits of agreement ( $95 \%$ confidence intervals of the differences) are given in Table I. The dioptric equivalent of these limits (calculated as $F=n-1 / r$, where $F$ is power, $n$ is the refractive index of cornea and $r$ is corneal

Table I. Pre-operative keratometry: the limits of agreement of repeated measurements with the same instrument, calculated as the largest difference $\pm 1.96 \mathrm{SD}$ of those differences

\begin{tabular}{lccc}
\hline $\begin{array}{l}\text { Instrument and } \\
\text { meridian }\end{array}$ & $\begin{array}{c}\text { Limit of } \\
\text { agreement } \\
\text { (mm) }\end{array}$ & Magnitude & $\begin{array}{c}\text { SRK II effect } \\
\text { (D) }\end{array}$ \\
\hline MK horizontal & -0.09 to 0.10 & 1.07 & 0.97 \\
MK vertical & -0.03 to 0.17 & 1.13 & 1.02 \\
AK horizontal & -0.08 to 0.24 & 1.71 & 1.54 \\
AK vertical & -0.01 to 0.13 & 0.80 & 0.72 \\
\hline
\end{tabular}

The dioptric equivalent of this limit and its effect on calculation of intraocular lens power have been calculated. $95 \%$ of results can be expected to differ by this amount or less.

MK, manual keratometry; AK, automated keratometry. 


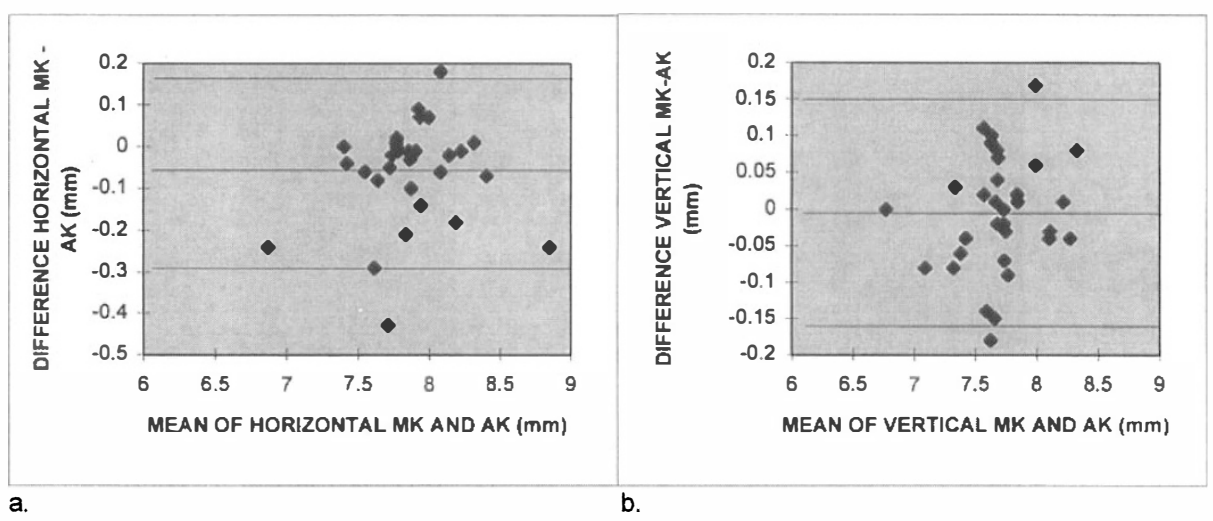

Fig. 2. Pre-operative keratometry: Difference between the means of three readings on the $M K$ and $A K$. The horizontal lines mark the mean difference and $\pm 1.96 S D$ of the differences (MK - AK). (a) Horizontal meridian. (b) Vertical meridian.

Table II. Pre-operative keratometry: the limits of agreement between manual keratometry and automated keratometry

\begin{tabular}{lccccc}
\hline & Limit of agreement & Magnitude & SRK II effect & \multicolumn{2}{c}{ No. outside limits } \\
\cline { 5 - 6 } Meridian & $(\mathrm{mm})$ & (D) & 2.56 & High & Low \\
\hline Horizontal & -0.29 to 0.17 & 1.76 & 2.30 & $1(3.1 \%)$ & $1(3.1 \%)$ \\
Vertical & -0.16 to 0.15 & 1.58 & $1(3.1 \%)$ & $1(3.1 \%)$ \\
\hline
\end{tabular}

The dioptric and SRK II equivalents have been calculated. As expected, less than $5 \%$ of differences lie outside the limits.

radius in metres) and its effect on the SRK II formula $(0.9 \times F$ in dioptres) are also given. Repeatability of measurement is similar for $\mathrm{MK}$ in both meridians and $\mathrm{AK}$ in the vertical meridian, but is poorer for $\mathrm{AK}$ in the horizontal meridian.

The comparison between MK and AK is shown in Fig. 2. The limits of agreement, with the number of observations falling outside those limits, are given in Table II, with the dioptric and SRK II equivalents of these ranges. The limit of agreement is widest for the horizontal meridian, with $95 \%$ of measurements with $\mathrm{MK}$ and $\mathrm{AK}$ expected to be within 2.56 dioptres of each other. The mean of the differences is a measure of the bias of the new test; mean horizontal corneal radius of curvature was $0.06 \mathrm{~mm}$ smaller with $\mathrm{MK}$ than AK, while mean vertical radius was $0.005 \mathrm{~mm}$ smaller with MK than AK.

\section{Post-operative Keratometry}

Mean-difference plots comparing MK and AK for assessment of suture-induced astigmatism (magnitude and axis of cylinder) are shown in Fig. 3. Limits of agreement are presented in Table III.

\section{DISCUSSION}

Keratometry is a means of estimating corneal radius of curvature and hence refractive power. As the true value for a given subject is not known a new keratometer can only be evaluated by comparison with an established machine. ${ }^{4}$ The repeatability of

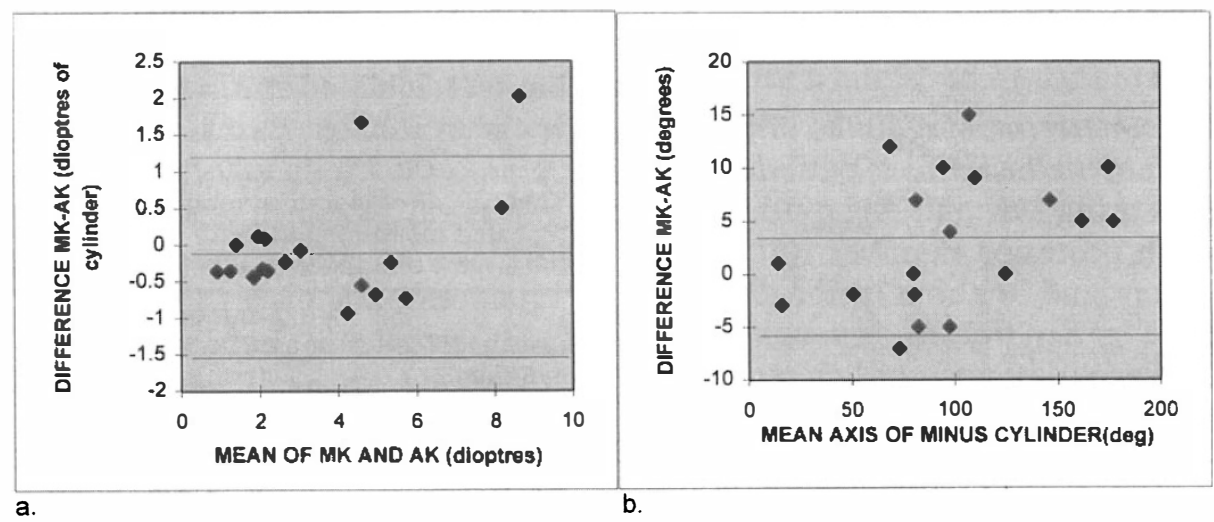

Fig. 3. Post-operative keratometry. (a) Differences in magnitude of cylinder. Note the increased spread with larger cylinders, indicating that agreement reduces as the cylinder magnitude increases. (b) Difference in axis of minus cylinder. The horizontal lines mark the mean difference $\pm 1.96 S D$ of the differences. 
Table III. Post-operative keratometry: the limits of agreement between manual keratometry and automated keratometry

\begin{tabular}{lcccc}
\hline & & \multicolumn{2}{c}{ No. outside limits } \\
\cline { 3 - 5 } Cylinder & Limit of agreement & Magnitude & High & Low \\
\hline Magnitude & -1.53 to $1.37 \mathrm{D}$ & $2.88 \mathrm{D}$ & $2(10 \%)$ & 0 \\
Axis & -5.97 to $15.45 \mathrm{deg}$ & $24.4 \mathrm{deg}$ & 0 & $1(5 \%)$ \\
\hline
\end{tabular}

More high outliers are present than would be expected. This is explained by examination of Fig. 3a, which shows increased spread with larger cylinders.

each method is important, as poor repeatability in either method will limit the extent to which agreement is possible. The limits of agreement calculated in this study do not allow a statement regarding a statistically significant difference between MK and $\mathrm{AK}$; rather the clinical significance of those limits needs to be examined.

In this study repeatability has been shown to be similar for MK and AK, although AK was more variable with readings along the horizontal meridian. The difference between MK and $\mathrm{AK}$ is also greatest with horizontal meridians, which is partly a reflection of this reduced repeatability. From Table I it can be seen that the power of an intraocular lens calculated using MK can be expected to vary by less than 1 dioptre in $95 \%$ of repeated measurements, while for AK the corresponding figure is 1.1 dioptres.

The difference between MK and AK can be expected to be larger than the difference between repeated readings using the same machine. From Table II, in $95 \%$ of cases $\mathrm{MK}$ and $\mathrm{AK}$ can be expected to generate lens powers within 1.94 dioptres of each other (the mean of the horizontal and vertical meridians). Examination of Fig. 2, however, shows that in most cases the agreement is much closer than this. The bias of AK (mean difference between $M K$ and $A K$ ) was small and not thought to be clinically significant.

Because the true corneal radius of curvature is not known it is not possible to determine which of the two machines is the less accurate. The poor repeatability of AK readings in the horizontal meridian may be explained in part by difficulties in holding the keratometer vertically, although the machine incorporates an alignment sensor that corrects for misalignment from the vertical of up to $15^{\circ}$.

The limits of agreement for post-operative assessment of suture-induced astigmatism are narrow enough to allow use of the $\mathrm{AK}$ as a guide to selective suture removal. The magnitude of the estimated cylinder differs considerably between the two machines. Agreement is poorest for high astigmatism (note the wider spread of points at higher mean cylinder in Fig. 3), and thus this difference is likely to be less clinically significant than the average disagreement would suggest.

The hand-held keratometer is quick and easy to use and can be operated with a minimum of training. Its compact size and self-contained operation make it useful when surgeries are held at multiple sites, avoiding the need for duplication of equipment. It is particularly useful for examination under anaesthetic, with children ${ }^{5}$ and with frail or bed-bound patients. It has been used with clinically satisfactory outcomes for pre-operative assessment of a small number of patients at the Spinal Injuries Unit at Stoke Mandeville Hospital (unpublished data). The handheld AK has been found to be difficult to use on neonates because of excessive eye movements, ${ }^{5}$ clearly this would not present a problem in patients under general anaesthetic.

MK has been shown by this study to have better repeatability than AK, possibly with clinically significant differences between them. For this reason the authors currently use MK for pre-operative evaluation of patients for cataract surgery. AK is used for the patient groups outlined above, when MK is technically difficult.

Keratometry accurately predicts which sutures are likely to cause refractive problems. ${ }^{2}$ It is used routinely in this hospital prior to refraction, thus avoiding the wasted refraction appointments that would otherwise occur when a patient is found to have astigmatism requiring suture removal. We consider the autokeratometer sufficiently reliable for this purpose.

The authors have no proprietary interest in any of the products used in this study.

The authors wish to thank Mr David Sculfor, Head Optometrist at Stoke Mandeville Hospital, for his assistance with this project.

Key words: Automated, Cataract, Keratometer, Repeatability.

\section{REFERENCES}

1. Sanders DR, Retzlaff J, Kraff MC. Comparison of the SRK II formula and other second generation formulae. $\mathrm{J}$ Cataract Refract Surg 1988;14:136-41.

2. Misson GP. Keratometry and post-operative astigmatism. Eye 1992;6:63-5.

3. Sunderraj P. Clinical comparison of automated and manual keratometry in pre-operative ocular biometry. Eye 1992;6:60-2.

4. Bland JM, Altman DG. Statistical methods for assessing agreement between two methods of clinical measurement. Lancet 1986;1:307-10.

5. Harvey EM, Miller JM, Dobson V. Reproducibility of corneal astigmatism measurements with a handheld keratometer in pre-school children. $\mathrm{Br} \mathrm{J}$ Ophthalmol 1995;79:983-90. 